EPJ Web of Conferences 59, 13003 (2013)

DOI: $10.1051 /$ epjconf/20135913003

(C) Owned by the authors, published by EDP Sciences, 2013

\title{
Spaced resolved analysis of suprathermal electrons in dense plasma
}

\author{
A. Moinard ${ }^{1,2, a}$, F. Petitdemange ${ }^{1}$, J. Larour ${ }^{3}$ and F.B. Rosmej ${ }^{1,2, b}$ \\ ${ }^{1}$ Sorbonne Université Pierre et Marie Curie, UMR 7065, LULI, case 128, 4 place Jussieu, \\ 75252 Paris Cedex 05, France \\ ${ }^{2}$ LULI, École Polytechnique, CEA, CNRS, équipe PAPD, 91128 Palaiseau, France \\ ${ }^{3}$ École Polytechnique, LPP, 91128 Palaiseau, France
}

\begin{abstract}
The investigation of the hot electron fraction is a crucial topic for high energy density laser driven plasmas: first, energy losses and radiative properties depend strongly on the hot electron fraction and, second, in ICF hohlraums suprathermal electrons preheat the D-T-capsule and seriously reduce the fusion performance. In the present work we present our first experimental and theoretical studies to analyze single shot space resolved hot electron fractions inside dense plasmas via optically thin X-ray line transitions from autoionizing states. The benchmark experiment has been carried out at an X-pinch in order to create a dense, localized plasma with a well defined symmetry axis of hot electron propagation. Simultaneous high spatial and spectral resolution in the X-ray spectral range has been obtained with a spherically bent quartz Bragg crystal. The high performance of the X-ray diagnostics allowed to identify space resolved hot electron fractions via the $\mathrm{X}$-ray spectral distribution of multiple excited states.
\end{abstract}

\section{INTRODUCTION}

Non-equilibrium electron statistics (or non-Maxwellian energy distribution functions) are at the center of worldwide research because they play an exceptional role for fusion research [1-5]: a) in the indirect drive scheme (hohlraum) of inertial fusion, hot electron production may lead to a preheat of the DT capsule and hinder subsequent compression above solid density to obtain ignition, b) in the direct drive scheme (fast ignition), hot electrons are created purposely by a PetaWatt laser via "hole boring" to initiate burn in the DT capsule, c) in atomic physics, suprathermal electrons lead to strong changes of almost all radiative properties like, e.g., ion charge state distributions, radiative losses, line intensities and line ratios. As plasma simulations of hot electrons are exceedingly difficult (and under continuous discussion) it is important to provide independent (from plasma simulations) data about suprathermal electrons. As high-resolution X-ray spectroscopy can provide a unique characterization of hot electrons [5], benchmark experiments are requested to validate non-Maxwellian atomic physics codes. An essential point of well characterized experiments is to have a well defined symmetry axis of hot electron propagation in dense plasmas. This is difficult to achieve in laser produced plasmas but can be quite well realized in $\mathrm{X}$-pinch experiments.

\footnotetext{
ae-mail: arnaud.moinard@upmc.fr

be-mail: frank.rosmej@upmc.fr
}

This is an Open Access article distributed under the terms of the Creative Commons Attribution License 2.0, which permits unrestricted use, distribution, and reproduction in any medium, provided the original work is properly cited. 


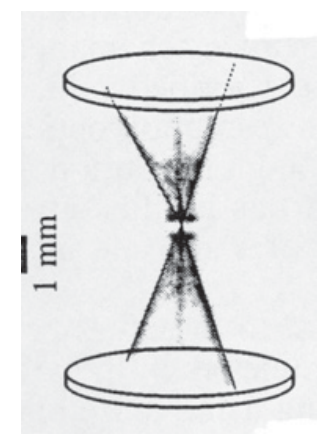

Figure 1. A schematic view of the X-Pinch.

\section{THE EXPERIMENTAL SETUP: X-PINCH}

An X-pinch principle is similar to those of the well known Z-pinch where plasma pressure is balanced by magnetic field pressure that is driven by a high current [6-9]. The X-pinch geometry is realized by crossing 2 or more wires - Fig. 1. At the crossing point, the magnetic pressure is highest and a hot dense plasma spot is formed.

The device used for the present experiments is a pulsed power generator developed for radiography needs $[10,11]$. The capacitors can deliver $850 \mathrm{~J}$ energy when charged with $40 \mathrm{kV}$. A peak current of $290 \mathrm{kA}$ can be obtained with a rise time of about $200 \mathrm{~ns}$. High purity $(99.99 \%)$ aluminum wires with $25 \mu \mathrm{m}$ diameter have been chosen.

In order to obtain space resolved information about the hot electron induced X-ray emission we implemented a spherically bend quartz crystal $(2 \mathrm{~d}=8.52$ Angstroem $)$ in the so called FSSR-2D scheme $[12,13]$. The distance between the source and the crystal was $40 \mathrm{~cm}$ which impose for the detector with the geometry to be at $117.7 \mathrm{~mm}$ from the crystal. As detector we used X-ray film: Kodak INDUSTREX 8800 , with a grain size of $4 \mu \mathrm{m}$. In order to have only the X-ray on the film, we add a filter made of $1 \mu \mathrm{m}$ of polpropylen covered by 100 angström of aluminum. This spectrometer setup allowed to record single shot high-resolution $\left(\frac{\lambda}{\Delta \lambda}=5000, \Delta z=40 \mu \mathrm{m}\right) \mathrm{X}$-ray images of the dense plasma emission.

\section{EFFECT OF HOT ELECTRONS ON LEVEL EXCITATION IN PLASMA}

Only well selected line emission is suitable for the analysis of hot electrons. In dense hot nonhomogenous plasmas X-ray emission of dielectronic satellites permits a unique characterization [5]. Of particular importance is that dielectronic statellite emission is not sensitive to radiative recombination (in contrast to resonance lines) confining their emission "naturally" to the dense hot plasma volume. In the present work we analyze the optically thin $\mathrm{He} \beta$ satellite emission $\left(1 s 2 l 3 l^{\prime}-1 s^{2} 2 l\right)$ with respect to its hot electron sensitivity. The principle idea is to separate in the highly resolved spectral distribution satellite transitions (about 60 E1-transitions originating from the 66 LSJ-split autoionizing levels $1 \mathrm{~s} 2131$ ') that are sensitive to different excitation channels:

a) Inner-shell excitation proceeds from the Li-like ions

$$
1 \mathrm{~s}^{2} 21+\mathrm{e}_{\text {hot }} \rightarrow 1 \mathrm{~s} 2131^{\prime}+\mathrm{e}
$$

b) dielectronic capture proceeding from the He-like ions.

The radiative transitions, the so-called dielectronic satellites (near $\mathrm{He} \beta$ ), are given by

$$
1 \mathrm{~s} 2131^{\prime} \rightarrow 1 \mathrm{~s}^{2} 21+h v_{\text {sat }}
$$




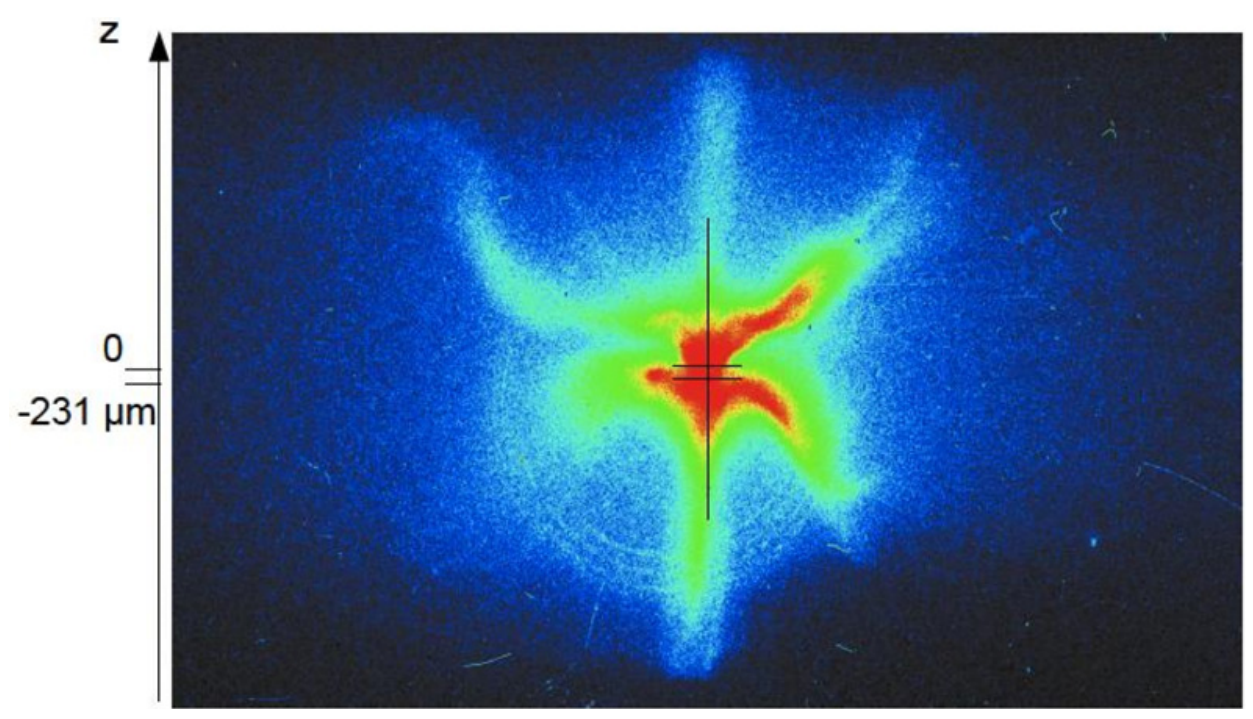

Figure 2. Pinhole image of the plasma, without any filter.

The inner-shell excitation channel is strongly enhanced by hot electrons whereas the dielectronic capture channel is only weakly perturbed. Hot electrons can therefore be characterized comparing the spectral distribution of both channels.

In dense plasmas, population can also be redistributed via electron collisions among different autoionizing states thereby allowing to detect density effects on the spectral distribution $[14,15]$.

\section{RESULTS}

Figure 2 shows the time integrated pinhole image: hot spot emission at the crossing point, emission from the heated wires and an emission along the z-direction.The z-direction corresponds to the propagation direction of the hot electrons that provoke emission when colliding with atoms and ions.

The Fig. 3 shows the $\mathrm{x}$-ray image of the spherical quartz spectrometer. Aluminum $\operatorname{He} \gamma, \operatorname{He} \beta$ and Ly $\alpha$ show faint emission in positive z-direction but very strong emission in negative z-direction, satellite emission, however, extends almost exclusively in the negative z-direction. Comparing Fig. 2 with Fig. 3 we suspect that hot electrons propagating in negative z-direction are responsible for the observed emission. Emission along the wavelengths axis (horizontal base line) with narrow spatial extension corresponds to the bremsstrahlung emitted from the hot spot (highest intensity).

The space resolved satellite emission shows important characteristic changes along the $\mathrm{z}$-axis, Fig. 4. Near the hot spot region (Fig. 4a) the $\beta 4$ transition is the most intense whereas far from the spot (Fig. 4b) $\beta 3$ is the most intense. The simulations show $[14,15]$ that the spectral distribution of Fig. $4 \mathrm{a}$ is almost close to the Boltzmann limit whereas a spectral distribution like in Fig. $4 \mathrm{~b}$ can neither be obtained for any realistic temperature and density changes.

\section{SIMULATIONS}

In order to study the spectral emission of dense plasmas containing hot electrons, we approximate the electron distribution function $\mathrm{F}(\mathrm{E})$ by [2]

$$
F(E)=\left(1-f_{\text {hot }}\right) \times F_{M}\left(E, T_{\text {bulk }}\right)+f_{\text {hot }} \times F_{M}\left(E, T_{\text {hot }}\right)
$$




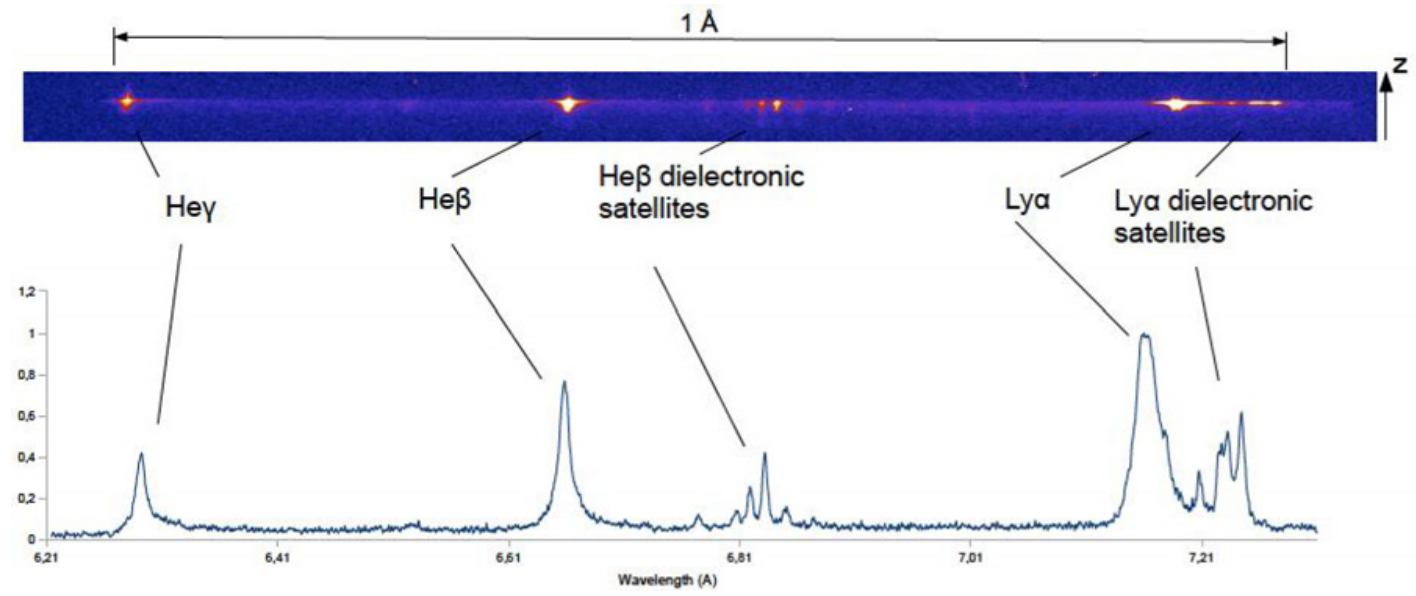

Figure 3. Image of the film, $\mathrm{z}$ corresponds to the spatial resolution and $\lambda$ to the spectral resolution.
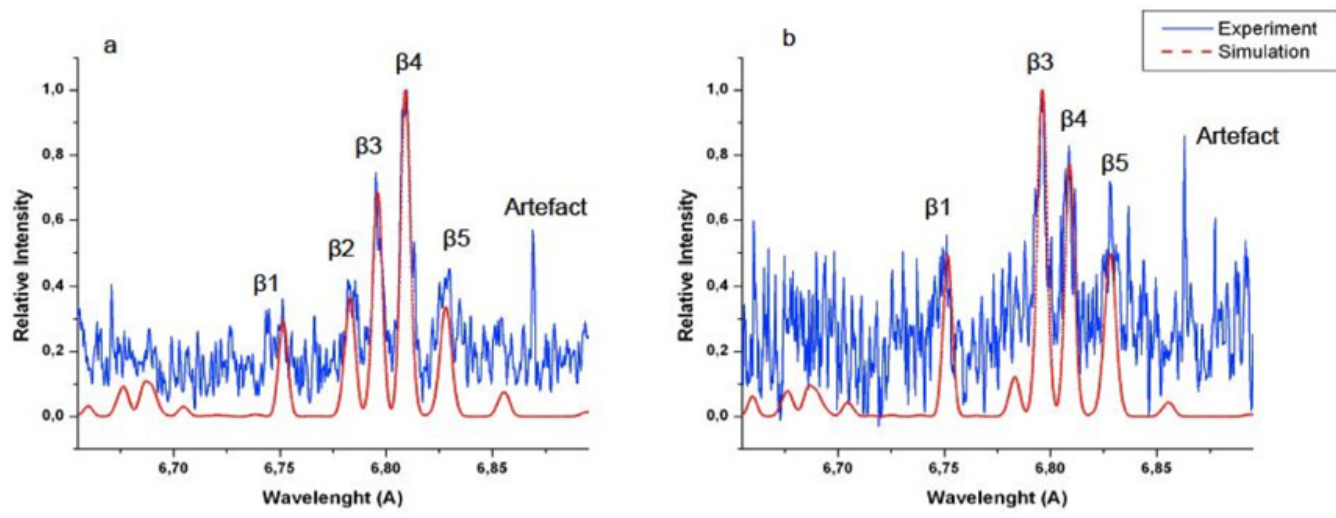

Figure 4. Space resolved spectral distribution of the $\mathrm{He} \beta$ satellite lines, a) $\mathrm{z}=0 \mu \mathrm{m}, \mathrm{b}) \mathrm{z}=-231 \mu \mathrm{m}$.

where $F_{M}(E, T)$ is a Maxwellian distribution function, $f_{\text {hot }}$ is the fraction of hot electrons characterized by a temperature $T_{\text {hot }}$. The generalized population matrix $W_{i j}$

$$
W_{i j}=A_{i j}+n_{e} \times C_{i j}+\Gamma_{i j}+n_{e} \times D C_{i j}+n_{e} \times I_{i j}+n_{e}^{2} \times T_{i j}+\ldots
$$

contains then the rate coefficients that are integrated over the distribution function given in eq. 3 . The atomic populations (ground states, single excited states as well as multiple excited autoionizing states) are given by a system of differential equations:

$$
\frac{d n_{i}}{d t}=-n_{i} \times \sum_{j} W_{i j}+\sum_{k} n_{k} \times W_{k i} .
$$

In order to compare the simulations directly with the observation, we calculate the spectral distribution according

$$
I=\sum_{i} \sum_{j>i} \frac{1}{4 \pi} \hbar \times \omega_{j i} \times n_{j} \times A_{j i} \times \varphi_{i j}
$$


where $i, j$ are the atomic levels, $n_{j}$ is the population of level $j, \hbar \times \omega_{i j}$ is the transition energy (transition from $j$ to $i, A_{j i}$ is the Einstein's coefficient of spontaneous emission and $\varphi$ is the line profile. Among other levels included in the simulation, we include exactly all LSJ-split 1s2131'-levels. Atomic data, cross sections and rate coefficients have been calculated with the FAC-code [16]. More details are described elsewhere [14].

As mentioned above, the simulations show that the spectral distribution (in particular the high emission of the $\beta 3$ satellite group) can only be reasonably described invoking hot electrons with a fraction of some percent: $f_{\text {hot }}=3 \%$. For the temperature $T_{\text {hot }}$, we have assumed $10 \mathrm{keV}$. Other parameters used in the simulation are: $T_{b u l k}=100 \mathrm{eV}$, an electronic density $n_{e}=10^{20} \mathrm{~cm}^{-3}$. The simulations of Fig. 4a indicate that the electronic density is about $n_{e}=10^{22} \mathrm{~cm}^{-3}$ indicating a spectral distribution close to a Boltzmann one, $T_{b u l k}=100 \mathrm{eV}$ and $f_{\text {hot }}=0$ was used in the simulation.

\section{CONCLUSION}

We have proposed the $\mathrm{He} \beta$ satellite emission as a sensitive diagnostic to the hot electron fraction. The method was successfully validated at an X-pinch facility with high-resolution X-ray spectroscopy. Hot electron fractions of some percent have be identified from the space resolved satellite spectral distribution far away from the central hot spot.

\section{References}

[1] J. D. Lindl, Phys. Plasmas 2, 3933 (1995)

[2] F.B. Rosmej, J. Phys. B. Lett. 30, L819 (1997)

[3] S.H. Glenzer, F.B. Rosmej, R.W. Lee, C.A. Back, K.G. Estabrook, B.J. MacCowan, T.D. Shepard, and R.E. Turner, Phys. Rev. Lett. 81, 365 (1998)

[4] F.B Rosmej, R. Schott, E. Galtier, P. Angelo, O. Renner, F.Y Khattak, V.S Lisitsa, and D. Riley, High Energy Density Physics 5, 191 (2009)

[5] F.B Rosmej, X-ray emission spectroscopy and diagnostics of non-equilibrium fusion and laser produced plasmas (editors Yaming Zou and Roger Hutton, Taylor and Francis, 2012, p. 267-341, ISBN: 9781420079043)

[6] W.H. Bennett, Phys. Rev. 45, 890 (1934)

[7] R.S. Pease, Proc. Phys. Soc. Lond. 70, 11 (1957)

[8] S.I. Braginskii, Sov. Phys. JETP 6, 494 (1958)

[9] M.G. Haines, Plasma Phys. Control. Fusion 53, 093001 (2001)

[10] J. Larour, L.E Aranchouk, and A.S Chuvatin, Transaction on PLasma Science 33, (2005)

[11] J. Larour and L.E. Aranchouk, Journal de Physique IV France 138, (2006)

[12] V.A. Boiko, A.V. Vinogradov, S.A. Pikuz, I.Yu. Skobelov, and A.Ya. Faenov, J. Sov. Las. Res. 6, 85 (1985)

[13] B.K.F Young, A.L. Osterheld, D.F. Price, R. Shepherd, R.E Stewart, A.Ya. Faenov, A.I Magunov, T.A. Pikuz, I.Yu. Skobolev, F. Flora, S. Bollanti, P. Di Lazzaro, T. Letardi, A. Grilli, L. Palladino, A. Reale and A. Scafati, and L. Reale, Review of Scientific Instruments 69, (1998)

[14] F. Petitdemange and F.B. Rosmej, Dielectronic satellites and Auger electron heating : irradiation of solids by intense XUV-Free Electron Laser radiation, New Trends in Atomic and Molecular Physics - Advanced Technological Applications, Springer 2013, editor M. Mohan, p. 1-28, in print

[15] F.B Rosmej and J.Jr Abdallah, Phys. Lett. A 245, 548 (1998)

[16] Ming Feng Gu, Canadian Journal of Physics 86, 675 (2008) 